\title{
Seismic interferometry: Reconstructing the earth's reflection response
}

\author{
Deyan Draganov ${ }^{1}$, Kees Wapenaar ${ }^{1}$, and Jan Thorbecke ${ }^{1}$
}

\begin{abstract}
In 1968, Jon Claerbout showed that the reflection response of a $1 \mathrm{D}$ acoustic medium can be reconstructed by autocorrelating the transmission response. Since then, several authors have derived relationships for reconstructing Green's functions at the surface, using crosscorrelations of (noise) recordings that were taken at the surface and that derived from subsurface sources.

For acoustic media, we review relations between the reflection response and the transmission response in $3 \mathrm{D}$ inhomogeneous lossless media. These relations are derived from a one-way wavefield reciprocity theorem. We use modeling results to show how to reconstruct the reflection response in the presence of transient subsurface sources with distinct excitation times, as well as in the presence of simultaneously acting noise sources in the subsurface. We show that the quality of reconstructed reflections depends on the distribution of the subsurface sources. For a situation with enough subsurface sources - that is, for a distribution that illuminates the subsurface area of interest from nearly all
\end{abstract}

directions - the reconstructed reflection responses and the migrated depth image exhibit all the reflection events and the subsurface structures of interest, respectively. With only a few subsurface sources, that is, with insufficient illumination, the reconstructed reflection responses are noisy and can even become kinematically incorrect. At the same time, however, the depth image, which was obtained from their migration, still shows clearly all the illuminated subsurface structures at their correct positions.

For the elastic case, we review a relationship between the reflection Green's functions and the transmission Green's functions derived from a two-way wavefield reciprocity theorem. Using modeling examples, we show how to reconstruct the different components of the particle velocity observed at the surface and resulting from a surface traction source. This reconstruciton is achieved using crosscorrelations of particle velocity components measured at the surface and resulting from separate P- and $\mathrm{S}$-wave sources in the subsurface.

\section{INTRODUCTION}

Seismic interferometry is the principle of generating new seismic records by crosscorrelating existing ones. This means that by taking a seismic record at an arbitrary point $\mathrm{A}$ and crosscorrelating it with another record at some point $\mathrm{B}$, one obtains a new seismic record at A as if it were from a source at B. This idea was first proposed by Claerbout (1968), who shows that the reflection response of an 1D medium can be synthesized from the autocorrelation of the transmission response measured at the surface. He names this method acoustic daylight imaging. Later, he conjectures that for 3D media, the crosscorrelation of the transmission responses measured at surface points A and B from ambient noise sources in the subsurface will reconstruct the reflection response at point $\mathrm{A}$ as if it were from a source at B.
The nature of the recorded fields at A and B can differ, as can the nature of the sources that causes those fields. The recorded wavefields can be diffuse as a result of multiple scattering at the medium heterogeneities or its boundaries (e.g., Lobkis and Weaver, 2001; Derode et al., 2003; van Tiggelen, 2003; and Snieder, 2004) or as a result of a random distribution of noise sources (e.g., Weaver and Lobkis, 2001; Wapenaar et al., 2002; Shapiro and Campillo, 2004; and Roux et al., 2005). Seismic interferometry using diffuse wavefields has been applied successfully for reconstruction of surface waves from seismological records (see, e.g., Campillo and Paul, 2003; Sabra et al., 2005; and Shapiro et al., 2005).

Recorded wavefields that can be used for seismic interferometry also can result from transient sources in a deterministic medium. In that case, the recorded fields at A and B can result from man-made sources such as the sources used for exploration, or from natural

Manuscript received by the Editor May 20, 2005; revised manuscript received January 15, 2006; published online August 17, 2006.

${ }^{1}$ Delft University of Technology, Department of Geotechnology, Mijnbouwstraat 120, 2628 RX Delft, The Netherlands. E-mails: d.s.draganov@citg. tudelft.nl; c.p.a.wapenaar@citg.tudelft.nl; j.w.thorbecke@citg.tudelft.nl.

(C) 2006 Society of Exploration Geophysicists. All rights reserved. 
sources like earthquakes (see, e.g., Schuster, 2001; Wapenaar et al., 2004; and Bakulin and Calvert, 2004, 2006).

In this paper, we limit ourselves to open deterministic media, in which we consider diffuse as well as deterministic fields. To record diffuse fields in open deterministic media, one needs noise sources. Using numerical modeling, Rickett and Claerbout (1996) and Rickett (1996) shows that to obtain a good reconstruction of the reflection response after the crosscorrelation of diffuse fields, one needs to have very long recording times and many spatially uncorrelated white-noise sources. The longer the recorded response, the better it approximates diffuse fields. At the same time, with longer recording times the response from more noise sources is recorded, and as a result, one obtains better illumination of the subsurface. For example, in the above-mentioned works for reconstruction of the surface waves, the recording times were of the order of weeks.
Contrary to the case with diffuse fields, when one uses deterministic fields in deterministic media, the seismic records need only be long enough to record the primaries and relevant multiples related to the deepest event of interest. This means, for the exploration case, a recording time of the order of seconds from each subsurface source. For such a case, one needs to record the response from many sources to obtain good illumination of the subsurface.

In this paper, we show how to reconstruct the reflection response at the free surface by crosscorrelating transmission responses of subsurface sources (transient, as well as noise) in acoustic and elastic media. For the acoustic case, we use relations between the reflection response and the transmission response, as presented in Wapenaar et al. (2002, 2004), where Claerbout's (1968) conjecture was proven for lossless 3D inhomogeneous media using a one-way wavefieldreciprocity theorem of the correlation type. We show how to reconstruct the reflection response for the case of separate recordings of the wavefields from each subsurface source (deterministic fields), as well as for the case in which uncorrelated subsurface sources act simultaneously (diffuse fields). For an accurate reconstruction of the reflection response, the recorded deterministic wavefields need to be decomposed into flux-normalized downgoing and upgoing components as a preprocessing step. With the help of numerically modeled data, we investigate the quality of the reconstructed reflection response and the reconstructed subsurface image for a varying number of noise sources present in the subsurface.

In the elastic case, we use relations such as those shown in Wapenaar and Fokkema (2006) in which the representations are derived using a two-way wavefield reciprocity theorem of the correlation type. We discuss modeling results of the reconstruction of the different components of the Green's function at the surface for the case in which we record the response of each subsurface $\mathrm{P}$ - and S-wave source separately. In practice, where the sources are of a mixed nature, we will need to perform the preprocessing step of decomposing the recorded wavefields into responses from $\mathrm{P}$ - and $\mathrm{S}$-wave sources.

\section{FROM RECORDED ACOUSTIC-TRANSMISSION DATA TO SIMULATED REFLECTION DATA}

Consider a lossless, source-free, 3D inhomogeneous domain D (see Figure 1a), embedded between the depth levels $\partial \mathrm{D}_{0}$ and $\partial \mathbb{D}_{1}$. Just above $\partial \mathbb{D}_{0}$ we have a free surface, and below $\partial \mathrm{D}_{1}$ the half-space is homogeneous. To this configuration we will apply a one-way reciprocity theorem of the correlation type. In general, a reciprocity theorem formulates a relation between two seismic states - for example, states $\mathcal{A}$ and $\mathcal{B}$. For states $\mathcal{A}$ and $\mathcal{B}$, we now take sources just above $\partial \mathrm{D}_{0}$ at points with coordinate vectors $\mathbf{x}_{A}$ and $\mathbf{x}_{B}$, respectively. Introducing these states into the one-way 
reciprocity theorem of the correlation type and making use of source-receiver reciprocity, we can write

$$
\begin{aligned}
R^{+}\left(\mathbf{x}_{A}, \mathbf{x}_{B}, t\right)+R^{+}\left(\mathbf{x}_{A}, \mathbf{x}_{B},-t\right) \\
=\delta\left(\mathbf{x}_{H, B}-\mathbf{x}_{H, A}\right) \delta(t) \\
\quad-\int_{\partial \mathrm{D}_{1}} T^{-}\left(\mathbf{x}_{A}, \mathbf{x}, t\right) * T^{-}\left(\mathbf{x}_{B}, \mathbf{x},-t\right) d^{2} \mathbf{x},
\end{aligned}
$$

where $R^{+}\left(\mathbf{x}_{A}, \mathbf{x}_{B}, t\right)$ is the flux-normalized one-way reflection response (upgoing reflection response) of the domain $\mathbb{D}$ observed at the surface at $\mathbf{x}_{A}$ from a source at the surface at $\mathbf{x}_{B} ; T^{-}\left(\mathbf{x}_{A}, \mathbf{x}, t\right)$ and $T^{-}\left(\mathbf{x}_{B}, \mathbf{x}, t\right)$ are the flux-normalized one-way transmission responses (upgoing transmission responses) of the domain $\mathrm{D}$ measured at $\mathbf{x}_{A}$ and $\mathbf{x}_{B}$, respectively, from a subsurface source at $\mathbf{x}$ on $\partial \mathrm{D}_{1} ; \delta()$ stands for the Dirac delta function; the asterisk * stands for the temporal convolution operation, and subscript $H$ means that only the horizontal coordinates from the vector are considered. Both the reflection response and the transmission response include all internal and freesurface multiples. Because here we are using decomposed wavefields, the superscript + in $R^{+}$means that the surface source emits downgoing waves, and the superscript - in $T^{-}$means that the subsurface source emits upgoing waves. In this way, equation 1 shows that the crosscorrelation of the transmission response measured at two surface points, $A$ and $B$, resulting from sources at depth level $\partial D_{1}$ in the subsurface, will reconstruct, with a minus sign, the reflection response plus its time reversal at $\mathrm{A}$ as if from an impulsive source at $\mathrm{B}$. Even though the relation is derived for subsurface sources at a constant depth level, the sources can be distributed randomly in depth because the crosscorrelation process eliminates the extra traveltimes. This can be shown more rigorously using the aforementioned derivation based on the two-way wavefield reciprocity.

In the following example, we show how to apply equation 1 in practice. Figure $1 \mathrm{~b}$ shows a 2D subsurface velocity model used for acoustic modeling, and $x_{1}$ and $x_{3}$ indicate coordinates in the horizontal direction and in the vertical direction, respectively. The model consists of four layers separated by one syncline and two dipping boundaries, respectively. Note that below the subsurface source level (represented by the random stars) there is an extra reflector, which does not comply with the assumption of a homogeneous half-space below the source level. The effects of this will be shown later. There are 561 receivers at the free surface $\left(x_{3}=0\right)$ at a lateral distance of $10 \mathrm{~m}$ from each other, starting at $x_{1}=1200 \mathrm{~m}$. The transient sources are situated between depth levels $x_{3}=700 \mathrm{~m}$ and $x_{3}=850 \mathrm{~m}$, with a random $x_{3}$ coordinate. In the horizontal direction the sources are spaced regularly at $25 \mathrm{~m}$, starting from $x_{1}=1200 \mathrm{~m}$ (in a total of 225 sources). The horizontal spread of the sources ensures sufficient illumination of the subsurface area of interest. The choice of the horizontal spacing stems from the fact that, ideally, we need to have at least two sources per wavelength to consider the source distribution to be continuous; in the following section we will show what happens when that condition is not fulfilled. For our configuration, we model a common source gather in a transmission geometry - that is, with a source in the subsurface and receivers at the free surface - for each source position, using a finite-difference modeling code.

Figure 1c shows the approximate acoustic subsurface model we used to test the robustness of the migration of the reconstructed reflection responses. The reflection responses were reconstructed from observed transmissions resulting from six subsurface sources. The first-layer velocity is presumed known and the other velocity values are $10 \%$ lower than the corresponding values in (Figure 1b). The boundaries are horizontal and at wrong depths.

Figure 1d shows the elastic subsurface model we used for generating transmission responses. White-noise sources (the grey stars) lie in the subsurface, with random $x_{3}$ coordinates between depth levels $x_{3}=700$ and $x_{3}=800 \mathrm{~m}$. In the horizontal direction, the sources are located every $21 \mathrm{~m}$ between $x_{1}=2100$ and $x_{1}=5691$. In this way, there are two sources per wavelength in the horizontal direction. The receivers at the surface are distributed every $15 \mathrm{~m}$ between $x_{1}=$ 2100 and $x_{1}=5700 \mathrm{~m}$.

Figure 2 shows an example of a common source gather in a transmission geometry for a subsurface source at the horizontal position $x_{1}=4000 \mathrm{~m}$. We extract one "master" trace from this panel (see Figure 3 ) and correlate it with each trace of the transmission panel. (A master trace is a trace at the position where, after crosscorrelation,

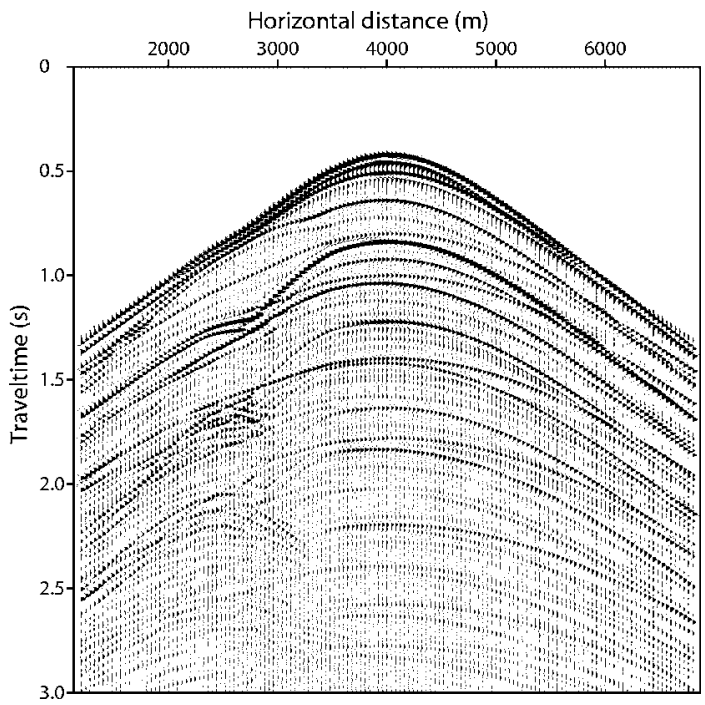

Figure 2. An acoustic transmission response for the model in Figure $1 \mathrm{~b}$, measured at the free surface, from a subsurface source with a horizontal coordinate of $x_{1}=4000 \mathrm{~m}$.

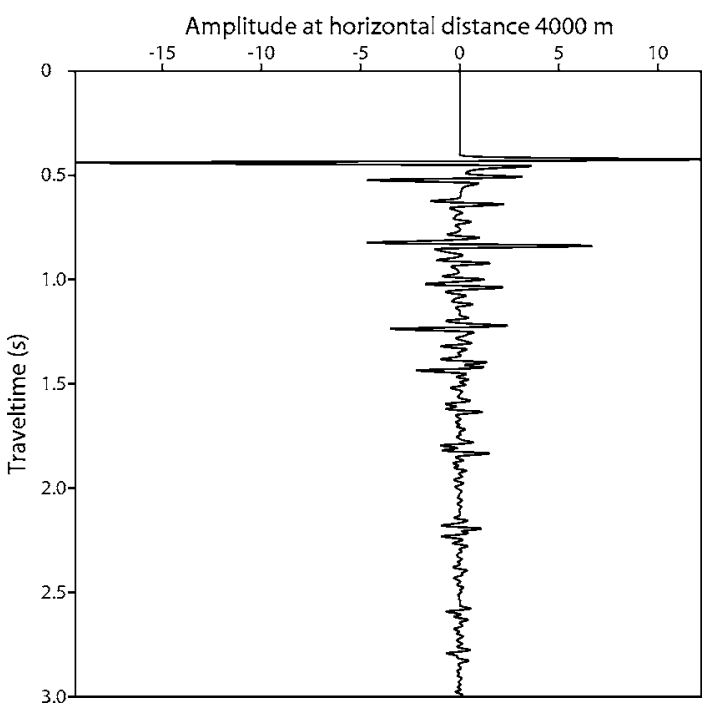

Figure 3. A transmission trace recorded at the free surface at the position $x_{1}=4000 \mathrm{~m}$ extracted from the panel shown in Figure 2 . 
we will obtain a simulated surface source.) The result of this operation represents the term $T^{-}\left(\mathbf{x}_{A}, \mathbf{x}, t\right) * T^{-}\left(\mathbf{x}_{B}, \mathbf{x},-t\right)$ in equation 1 for variable $\mathbf{x}_{A}$ and for fixed $\mathbf{x}_{B}$ and $\mathbf{x}$. We perform the same procedure for all modeled common source gathers in transmission geometries and sum the individual results. The sum represents the integral along $\mathbf{x}$ in equation 1 for variable $\mathbf{x}_{A}$ and fixed $\mathbf{x}_{B}$.

The output of the above procedure contains events at positive and at negative times. For an ideal distribution of the subsurface sources, the reflection response and its time reversal - that is, the reflection responses at positive and negative times — give us redundant information. Because the reflection response is a causal function of time, we have simply muted the negative times to obtain the final result in Figure 4.

Comparing the reconstructed reflection response from Figure 4 with the directly modeled reflection response in Figure 5, we see that kinematically the two data panels are the same except for the noisier character of the reconstructed reflection panel. Primaries, and multiple arrivals are reconstructed correctly. Equation 1 was derived with the assumption that the medium below the subsurface source level is homogeneous. Wapenaar and Fokkema (2006) showed theoretically that the presence of inhomogeneities below the source level results in an extra error term (ghosts) on the left-hand side of relation 1. Draganov et al. (2004) demonstrated the appearance of those ghosts with numerical modeling. They also showed that such ghost events are greatly weakened (and even can disappear) when the subsurface sources are distributed randomly in depth. That weakening results from the incoherent summation in equation 1 of the individual contributions to these ghost events.

In Figure 4, the ghost event with an apex around $0.2 \mathrm{~s}$ is strongly weakened in its lateral continuity. Because of this, it is not easy to compare that ghost event's amplitude with the amplitude of the (real) first arrival with an apex at $0.39 \mathrm{~s}$. However, at its maximum amplitude the ghost event is about four times weaker. The incoherent summation is also the cause of the noisier nature of the reconstructed reflection response panel above the first arrival. On the other hand, the

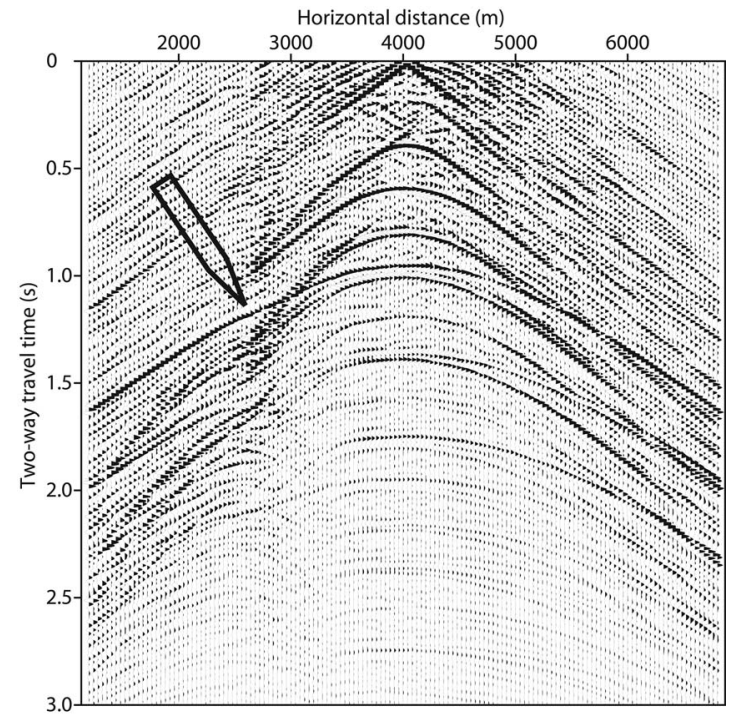

Figure 4. A reconstructed reflection response for the model in Figure $1 \mathrm{~b}$, as if from a source at $\left(x_{1}, x_{3}\right)=(4000,0) \mathrm{m}$. All primary reflections and multiply reflected arrivals are reconstructed correctly. The arrow points at the reflection event from the layer boundary below the sources. real reflection arrivals from the layer boundary below the sources are reconstructed correctly after crosscorrelation (see the events indicated by the arrows in Figures 4 and 5).

According to equation 1, to reconstruct the reflection response at the surface we must have separate recordings of the transmission response from each of the transient subsurface sources. In practice, it is not always easy to find sources acting at distinct times and allowing separate transmission measurements. Assuming the subsurface sources to be uncorrelated in time, we can rewrite equation 1 as

$$
\begin{aligned}
& R^{+}\left(\mathbf{x}_{A}, \mathbf{x}_{B}, t\right)+R^{+}\left(\mathbf{x}_{A}, \mathbf{x}_{B},-t\right) \\
& \quad=\delta\left(\mathbf{x}_{H, B}-\mathbf{x}_{H, A}\right) \delta(t)-T_{o b s}^{-}\left(\mathbf{x}_{A}, t\right) * T_{o b s}^{-}\left(\mathbf{x}_{B},-t\right),
\end{aligned}
$$

where $T_{\text {obs }}^{-}\left(\mathbf{x}_{A}, t\right)$ and $T_{\text {obs }}^{-}\left(\mathbf{x}_{B}, t\right)$ represent the transmission responses recorded at the surface at $\mathbf{x}_{A}$ and $\mathbf{x}_{B}$, respectively. The responses were generated by simultaneously acting white-noise sources in the subsurface.

Again, we use the subsurface model from Figure 1b. In the presence of many subsurface white-noise sources acting simultaneously, the transmission response $T_{\text {obs }}^{-}\left(\mathbf{x}_{A}, t\right)$ recorded at the surface (for variable $\mathbf{x}_{A}$ ) will look like the one in Figure 6 . In Figure 6 , we show only the initial $3 \mathrm{~s}$ of the entire modeled transmission recording, which is 23 minutes long. Because the subsurface sources act simultaneously, each trace from this recording already contains in itself the sum of all present subsurface sources. According to equation 2, to reconstruct the reflection response we only need to extract one master trace from the transmission panel (as in the example in Figure 7) and correlate it with all traces of the panel. The reconstructed reflection response, is shown in Figure 8.

Equations 1 and 2 give us a means for reconstructing the reflection response in two different ways, depending on the practical situation. When we can record at the surface the separate transmission responses from each of the transient subsurface sources, for example, from different earthquakes, we can use equation 1. However, when we do not know exactly when the subsurface sources will be active,

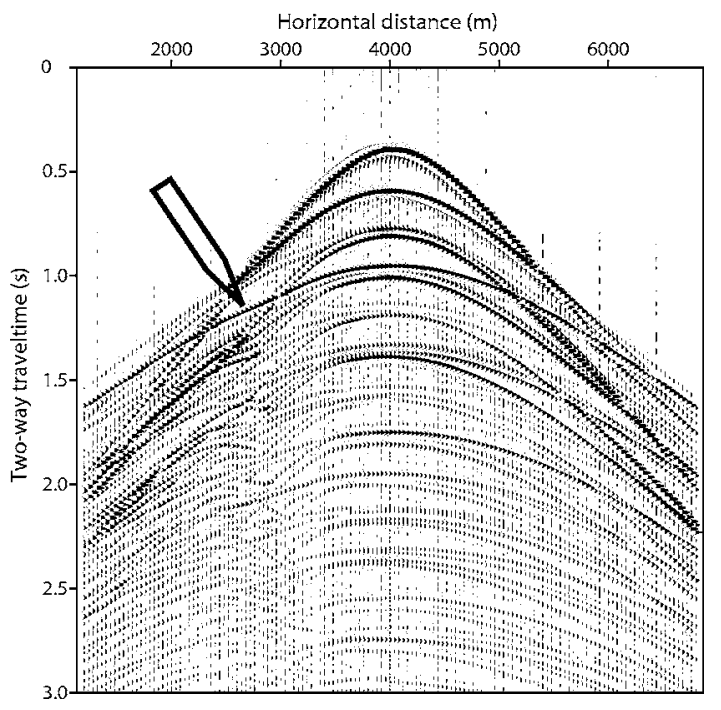

Figure 5. A directly modeled reflection response for the model in Figure $1 \mathrm{~b}$, from a source at $\left(x_{1}, x_{3}\right)=(4000,0)$. The arrow points at the reflection event from the layer boundary below the sources. 
or when the subsurface sources emit noise continuously, we can just record the upcoming waves at the surface for some period of time and then use equation 2. Of course, in that case the quality of the reconstructed reflection response we obtain will depend on how uncorrelated the noise sources are. As a result, we need a very long recording time (hours, days, or even weeks, depending on the situation). This is in contrast to the short recording times that we need when we are using equation 1 . The latter times need be only long enough to record the primaries and relevant multiples related to the deepest event of interest - that is, on the order of seconds.

The reconstruction procedure using either equation 1 or equation 2 depends on the distribution of the subsurface sources. Randomness in the vertical distribution of the subsurface sources will weaken the requirement to have no reflectors below the sources. To reconstruct all the reflection events of interest, we use enough subsurface sources to illuminate the considered part of the subsurface from all the necessary directions. In the following section, we investigate the impact on the reconstruction process when that condition is not fulfilled.

\section{FROM SIMULATED ACOUSTIC-REFLECTION DATA TO A DEPTH IMAGE}

Once we have the reconstructed reflection responses, we can image the subsurface using a standard poststack or prestack migration procedure. However, Artman et al. (2004) proposed an alternative in which depth image of the subsurface can be constructed by applying shot-profile migration - which also is a crosscorrelation process directly to the noise-transmission responses recorded at the surface. In that paper, we proved this idea mathematically. The proof is as follows. We start with a relation in the frequency domain for downward extrapolation of the reflection response

$$
\begin{aligned}
\hat{R}^{+}\left(\boldsymbol{\xi}_{A}, \boldsymbol{\xi}_{B}, \omega\right)= & \int_{\partial \mathrm{D}_{0}} \int_{\partial \mathrm{D}_{0}}\left\{\hat{W}^{+}\left(\boldsymbol{\xi}_{A}, \mathbf{x}_{A}, \omega\right)\right\}^{*} \hat{R}^{+}\left(\mathbf{x}_{A}, \mathbf{x}_{B}, \omega\right) \\
& \times\left\{\hat{W}^{-}\left(\mathbf{x}_{B}, \boldsymbol{\xi}_{B}, \omega\right)\right\}^{*} d^{2} \mathbf{x}_{A} d^{2} \mathbf{x}_{B} .
\end{aligned}
$$

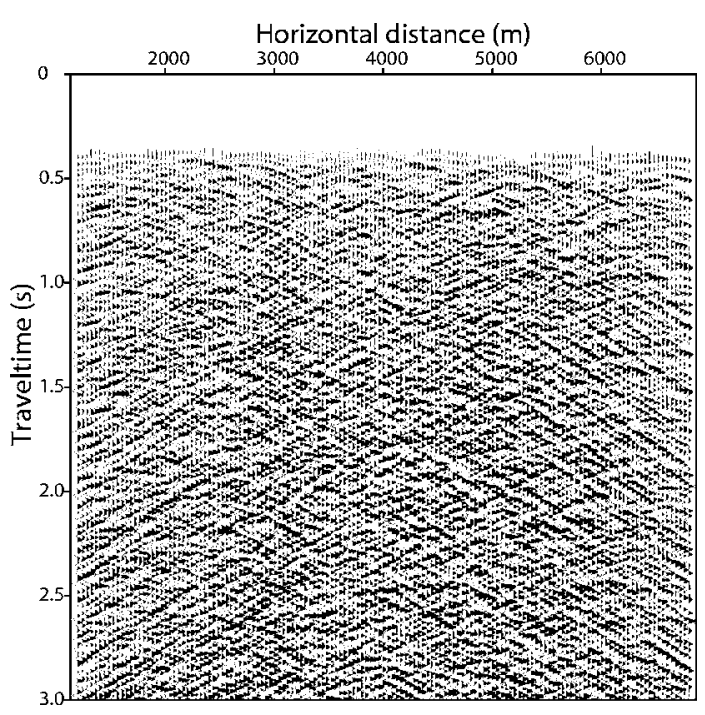

Figure 6 . The first $3 \mathrm{~s}$ of a 23-minute-long acoustic-transmission response for the model in Figure 1b, from 225 white-noise sources acting simultaneously.
Here, $\hat{W}^{+}\left(\boldsymbol{\xi}_{A}, \mathbf{x}_{A}, \omega\right)$ and $\hat{W}^{-}\left(\mathbf{x}_{B}, \boldsymbol{\xi}_{B}, \omega\right)$ are the one-way wavefield extrapolation operators. The superscript $*$ denotes complex conjugation, which turns the forward extrapolation operators into approximate inverse operators; $\hat{R}^{+}\left(\boldsymbol{\xi}_{A}, \boldsymbol{\xi}_{B}, \omega\right)$ represents the downward-extrapolated reflection response, with $\boldsymbol{\xi}_{A}$ and $\boldsymbol{\xi}_{B}$ representing coordinate vectors at a depth level in the subsurface. Substituting the frequency domain equivalent of equation 2 , using reciprocity of the extrapolation operator $\hat{W}^{-}=\hat{W}^{+}$and the fact that the free-surface reflection coefficient is given by $r=-1$, it follows that the reflection response $R^{+}\left(\boldsymbol{\xi}_{A}, \boldsymbol{\xi}_{B}, \omega\right)$ in the subsurface can be written as

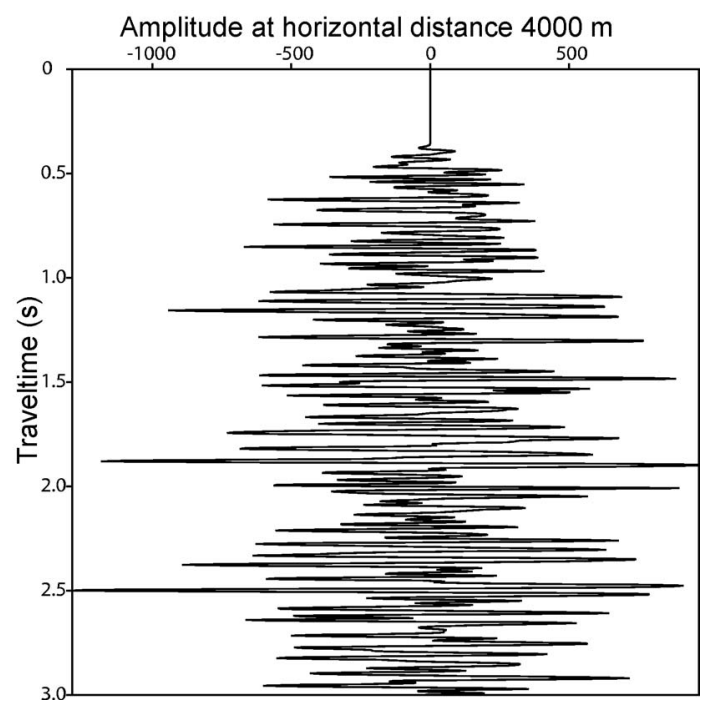

Figure 7. A transmission trace recorded at the free surface at position $x_{1}=4000 \mathrm{~m}$ extracted from the panel shown in Figure 6 .

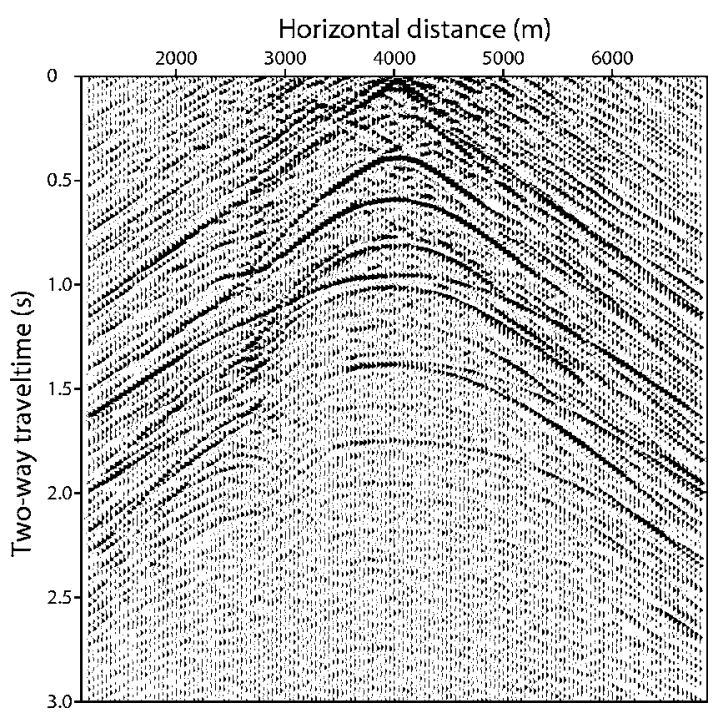

Figure 8. A reconstructed reflection response for the model in Figure $1 \mathrm{~b}$, as if from a source at $\left(x_{1}, x_{3}\right)=(4000,0)$. This reflection response was obtained by correlating the trace from Figure 7 with the transmission panel in Figure 6 . The primary reflection and the multiply reflected arrivals are reconstructed correctly. 


$$
\begin{aligned}
\hat{R}^{+}\left(\boldsymbol{\xi}_{A}, \boldsymbol{\xi}_{B}, \omega\right)= & \int_{\partial \mathrm{D}_{0}}\left\{\hat{W}^{+}\left(\boldsymbol{\xi}_{A}, \mathbf{x}_{A}, \omega\right)\right\}^{*} \hat{T}_{o b s}^{-}\left(\mathbf{x}_{A}, \omega\right) d^{2} \mathbf{x}_{A} \\
& \times\left\{\int_{\partial \mathrm{D}_{0}} \hat{W}^{+}\left(\boldsymbol{\xi}_{B}, \mathbf{x}_{B}, \omega\right) r \hat{T}_{o b s}^{-}\left(\mathbf{x}_{B}, \omega\right) d^{2} \mathbf{x}_{B}\right\} \\
& + \text { acausal terms. }
\end{aligned}
$$

The first integral describes inverse extrapolation of the transmission response $\hat{T}_{o b s}^{-}\left(\mathbf{x}_{A}, \omega\right)$ to $\boldsymbol{\xi}_{A}$; the second integral describes forward extrapolation of the downward-reflected transmission response $r \hat{T}_{o b s}^{-}\left(\mathbf{x}_{B}, \omega\right)$ to $\boldsymbol{\xi}_{B}$. The product of the first integral and the complex conjugate of the second integral corresponds to a crosscorrelation of the extrapolated responses at $\boldsymbol{\xi}_{A}$ and $\boldsymbol{\xi}_{B}$. In addition, if we take $\boldsymbol{\xi}_{A}$ equal to $\boldsymbol{\xi}_{B}$ and sum over all frequency components, we obtain the zero-offset image of the subsurface at this level.

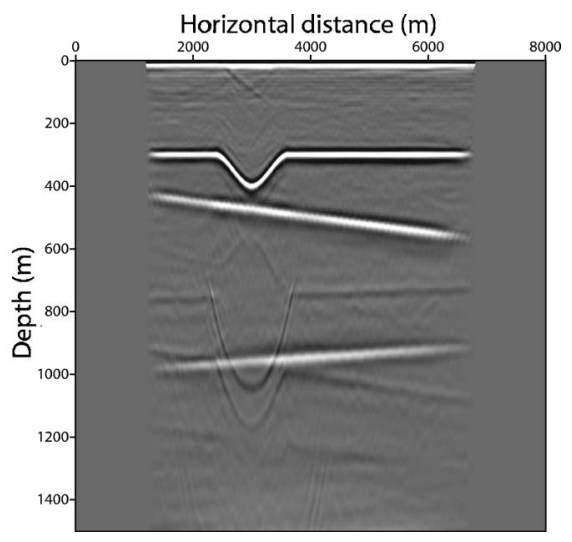

Figure 9. A depth image from reconstructed reflection responses at each simulated source position at the surface, in the presence of 225 subsurface white-noise sources. All the subsurface reflectors are imaged.
Apparently, the results from direct migration of the transmission responses and from migration of the reconstructed reflections are identical. The choice of which to use depends on our goals. If we have a velocity model of the subsurface, we can migrate the transmission responses immediately-that is, we can migrate the data [see also Artman (2006)]. When we have no velocity information of the subsurface, we first should reconstruct the reflection responses and apply velocity analysis.

Let us reconstruct the reflection responses for all the simulated surface source positions, that is, for all geophone positions, as we did for the simulated source at $x_{1}=4000 \mathrm{~m}$ (see Figure 8). Instead of performing velocity analysis to estimate the velocity structure of the subsurface, as we would do with real data, for the moment we use the exact velocity model with the idea of emphasizing the characteristics of the reconstructed depth images. For the same reason, we do not apply any multiple-elimination scheme. To obtain the depth image, we used a prestack shot-profile migration (Claerbout, 1971). The resulting depth image is shown in Figure 9.

We can appreciate the quality of the depth image because all three reflectors are imaged. The event at depth $x_{3}=0$ is a result of the (band-limited) delta functions on the simulated reflection panels. The other events imaged are consequences of the free-surface and internal multiples in the reconstructed reflection responses.

Of course, in practice we do not always have enough subsurface sources. With the following examples, we investigate how the reconstructed reflection responses change when the number of subsurface sources is reduced. We also investigate the changes in the migrated images obtained from these reconstructed reflection responses. Again, we use the subsurface model from Figure 1b, but this time we record at the free surface the transmission responses from, respectively, 37 and six white-noise sources. In these cases, in the horizontal direction there is one source per three or per 20 wavelengths, respectively. The sources are distributed randomly in the vertical $\left(x_{3}\right)$ direction, and they are distributed regularly in the horizontal $\left(x_{1}\right)$ direction. Figures 10a and 11a show the reconstructed reflection responses for the above-mentioned numbers of sources. We see that when the number of subsurface sources decreases, that is, when the part of the subsurface we are interested in is illuminated from fewer angles, the overall reconstructed reflection response becomes noisier and later arrivals get obscured. In the extreme case of six sources, the reconstructed events are kinematically incorrect.

For the same cases of 37 and six subsurfacenoise sources, we reconstruct the simulated reflection response panels for each simulated surface source position and migrate them to obtain the images shown in Figures $10 \mathrm{~b}$ and $11 \mathrm{~b}$, respectively. Comparing them and the image in Figure 9 with the model in Figure 1b, we see that the subsurface model is reconstructed accurately even for the case with only six subsurface sources.

This accuracy of the subsurface image is explained as follows. In the integration process, after correlation, the main contributions to the reconstructed responses come from the Fresnel zones around the stationary phase points. In reconstruction of the reflection responses, there is only one integral along sources in the subsurface (equation 1). With sparse source sampling, the stationary point is easily missed. On the other 
hand, migration involves additional integrations along the surface (equation 4). With good receiver sampling, generally the stationary points are covered by the integrals.

In the examples, we also see that when the number of subsurface sources decreases, the migrated images begin to show an extra reflectorlike feature close to the surface. This feature is a consequence of the ghost reflection event caused by the reflector below the sources. With only six subsurface sources, the vertical positions of the subsurface sources lack the necessary randomness to suppress this type of ghost event.

To demonstrate that the good quality of the obtained images is not purely a result of migration with the exact velocity model, Figure 12 shows the result of migrating the reconstructed reflection responses for the case of six subsurface sources using the approximate velocity model in Figure 1c. Here, we presume that we know the exact velocity of the first layer, whereas the velocities of the other layers are $10 \%$ lower than the ones used in Figure 1b. The layer boundaries are taken horizontally and at incorrect depths. As Figure 12 shows, migration of reflection responses reconstructed from correlations behaves similarly to migration of normal reflection data: All the reflectors are imaged, although at wrong positions and with artifacts.

\section{FROM RECORDED ELASTIC TRANSMISSION DATA TO SIMULATED REFLECTION DATA}

For the elastic case, Wapenaar and Fokkema (2006) used a two-way wavefield reciprocity theorem to derive an exact expression for the Green's function $G_{p, q}^{v, f}\left(\mathbf{x}_{A}, \mathbf{x}_{B}, t\right)$. Their Green's function represents the observed particle velocity $(v)$ in the $x_{p}$-direction $(p=1,2,3)$ at a point with coordinate vector $\mathbf{x}_{A}$ and resulting from an impulsive force source $(f)$ in the $x_{q}$-direction at $\mathbf{x}_{B}$. This Green's function can be expressed in terms of a crosscorrelation of Green's functions $G_{p, K}^{v, \phi}\left(\mathbf{x}_{A}\right.$, $\mathbf{x}, t)$ and $G_{q, K}^{v, \phi}\left(\mathbf{x}_{B}, \mathbf{x}, t\right)$ that represent the observed
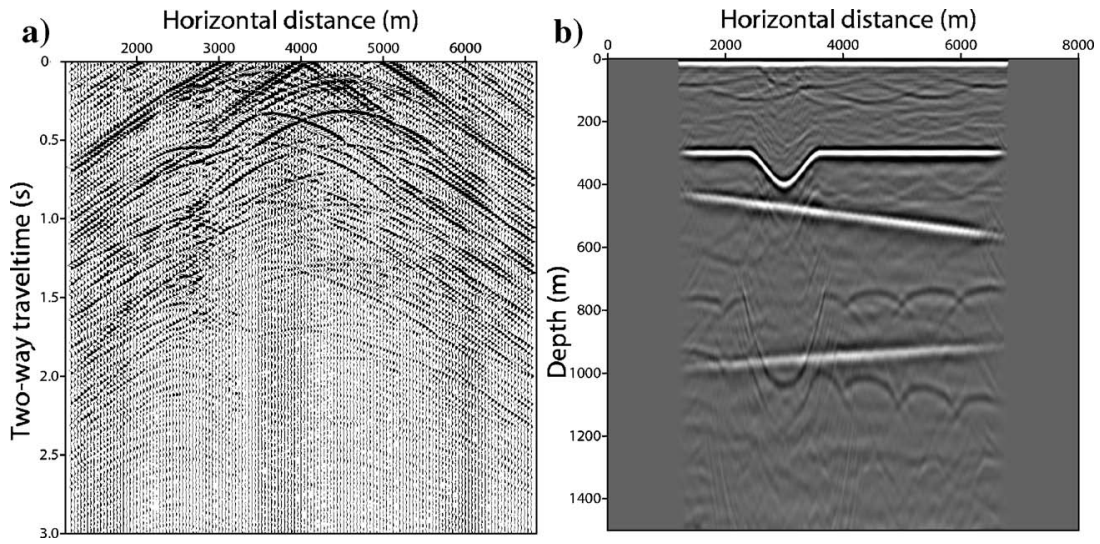

Figure 11. (a) A reconstructed reflection response for the model in Figure 1b, as if from an implusive source at $\left(x_{1}, x_{3}\right)=(4000,0)$. This time, there were six white-noise sources in the subsurface, distributed randomly in the vertical direction. The distance between the white-noise sources in the horizontal direction is $1000 \mathrm{~m}$. The reconstructed reflection events are kinematically incorrect. (b) A depth image from reconstructed reflection responses. The migrated image still shows all the reflectors.
When the observation points $\mathbf{x}_{A}$ and $\mathbf{x}_{B}$ are chosen at the free surface, the integration over the closed boundary $\partial \mathrm{D}$ can be replaced by an integration over some open boundary $\partial \mathbb{D}_{1}$ in the subsurface. Hence,

$$
\begin{aligned}
& G_{p, q}^{v, \tau}\left(\mathbf{x}_{A}, \mathbf{x}_{B}, t\right)+G_{p, q}^{v, \tau}\left(\mathbf{x}_{A}, \mathbf{x}_{B},-t\right) \\
& \quad \approx \frac{2}{\rho c^{K}} \int_{\partial \mathrm{D}_{1}} G_{p, K}^{v, \phi}\left(\mathbf{x}_{A}, \mathbf{x}, t\right) * G_{q, K}^{v, \phi}\left(\mathbf{x}_{B}, \mathbf{x},-t\right) d^{2} \mathbf{x}
\end{aligned}
$$

where $G_{p, q}^{v, \tau}\left(\mathbf{x}_{A}, \mathbf{x}_{B}, t\right)$ represents the observed particle velocity $(v)$ in the $x_{p}$-direction at $\mathbf{x}_{A}$ at the free surface and resulting from an impulsive traction source $(\tau)$ in the $x_{q}$-direction at $\mathbf{x}_{B}$ at the free surface. Thus, this Green's function represents the reflection response; the Green's functions $G_{p, K}^{v, \phi}\left(\mathbf{x}_{A}, \mathbf{x}, t\right)$ and $G_{q, K}^{v, \phi}\left(\mathbf{x}_{B}, \mathbf{x}, t\right)$ now also are recorded at the free surface and represent transmission responses.

Figure $1 \mathrm{~d}$ shows the 2D elastic subsurface model, which we use to particle velocities $(v)$ in the $x_{p}$ - and $x_{q}$-directions, at $\mathbf{x}_{A}$ and $\mathbf{x}_{B}$, respectively. Those observed particle velocities result from impulsive sources at points with coordinate vectors $\mathbf{x}$ along an arbitrarily shaped closed boundary $\partial \mathbb{D}$. The superscript $\phi$ stands for P-wave sources (when $K=0$ ) and for S-wave sources with different polarizations (when $K=1,2,3$ ). To obtain separate $\mathrm{P}$ - and S-wave source responses we will need to perform wavefield decomposition at the source level as preprocessing. The following relation was obtained after several approximations that mainly concern the amplitude:

$$
\begin{aligned}
G_{p, q}^{v, f}\left(\mathbf{x}_{A}, \mathbf{x}_{B}, t\right)+G_{p, q}^{v, f}\left(\mathbf{x}_{A}, \mathbf{x}_{B},-t\right) \\
\quad \approx \frac{2}{\rho c^{K}} \oint_{\partial \mathrm{D}} G_{p, K}^{v, \phi}\left(\mathbf{x}_{A}, \mathbf{x}, t\right) * G_{q, K}^{v, \phi}\left(\mathbf{x}_{B}, \mathbf{x},-t\right) d^{2} \mathbf{x} .
\end{aligned}
$$

Note that Einstein's summation convention over repeated indices applies only to the subscripts. At and outside $\partial \mathrm{D}$, the medium is assumed to be homogeneous and isotropic. In equation $5, c^{K}$ stands, respectively, for the P-wave velocity $\left(c_{p}\right)$ at and outside $\partial \mathrm{D}$ when $K$ $=0$, and for the S-wave velocity $\left(c_{s}\right)$ at and outside $\partial \mathrm{D}$ when $K$ $=1,2,3$. Finally, $\rho$ is the density at and outside $\partial \mathbb{D}$. 


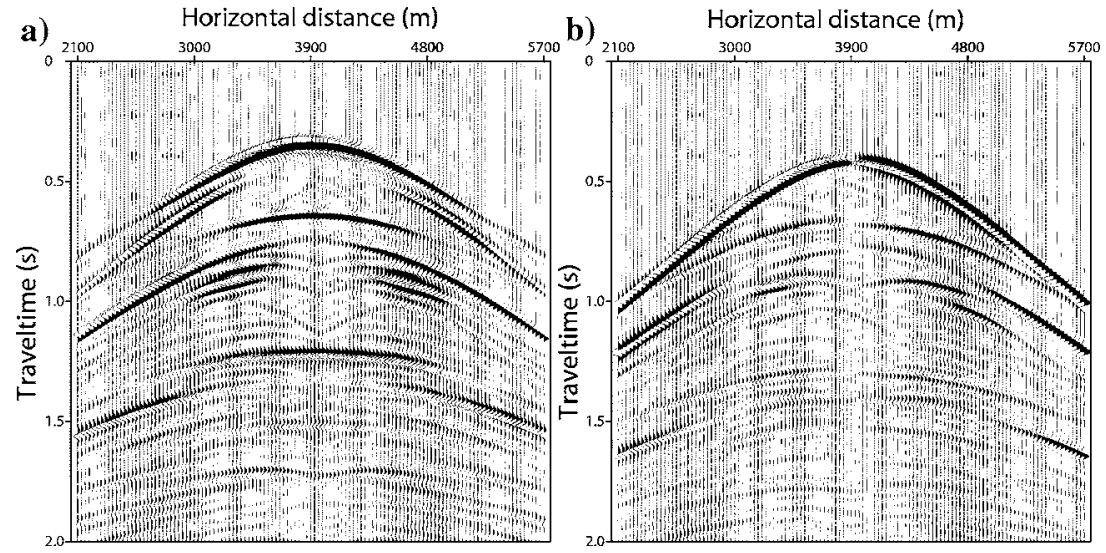

Figure 13. (a) A recorded vertical particle velocity $G_{3,0}^{v, \phi}\left(\mathbf{x}_{A}, \mathbf{x}, t\right)$ resulting from a P-wave source at $\left(x_{1}, x_{3}\right)=(748,3906) \mathrm{m}$ for the model in Figure 1d. (b) Recorded vertical particle velocity $G_{3,2}^{v,}\left(\mathbf{x}_{A}, \mathbf{x}, t\right)$ resulting from an $\mathrm{SV}$-wave source at $\left(x_{1}, x_{3}\right)=(748,3906) \mathrm{m}$ for the model in Figure 1d.
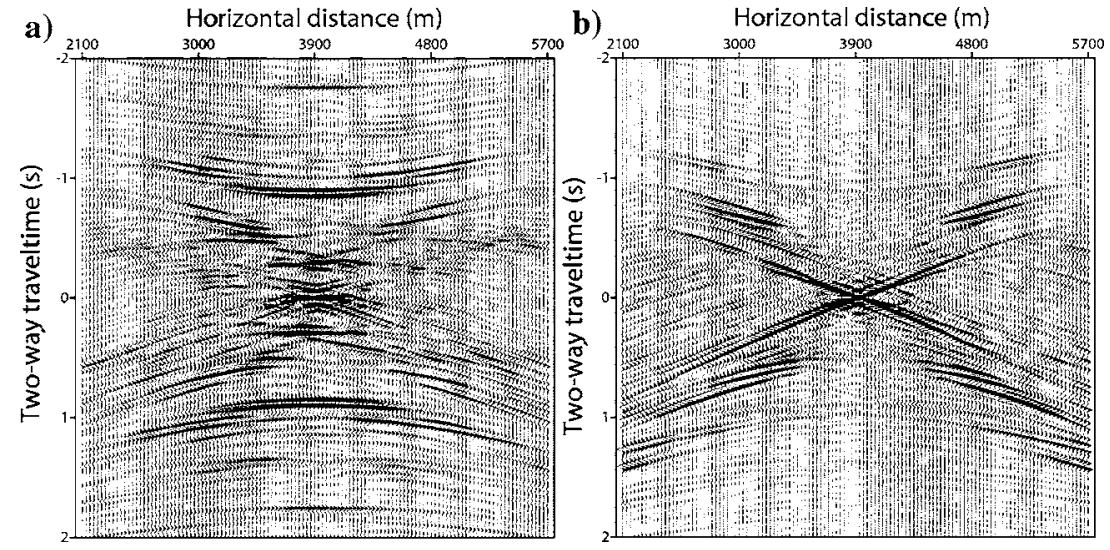

Figure 14. (a) The result from the correlation and integration in the right-hand side of equation 6 for P-wave subsurface sources $(K=0)$ for variable $\mathbf{x}_{A}$ and fixed $\mathbf{x}_{B}$. (b) A similar situation to (a), but here for SV-wave subsurface sources $(K=2)$.
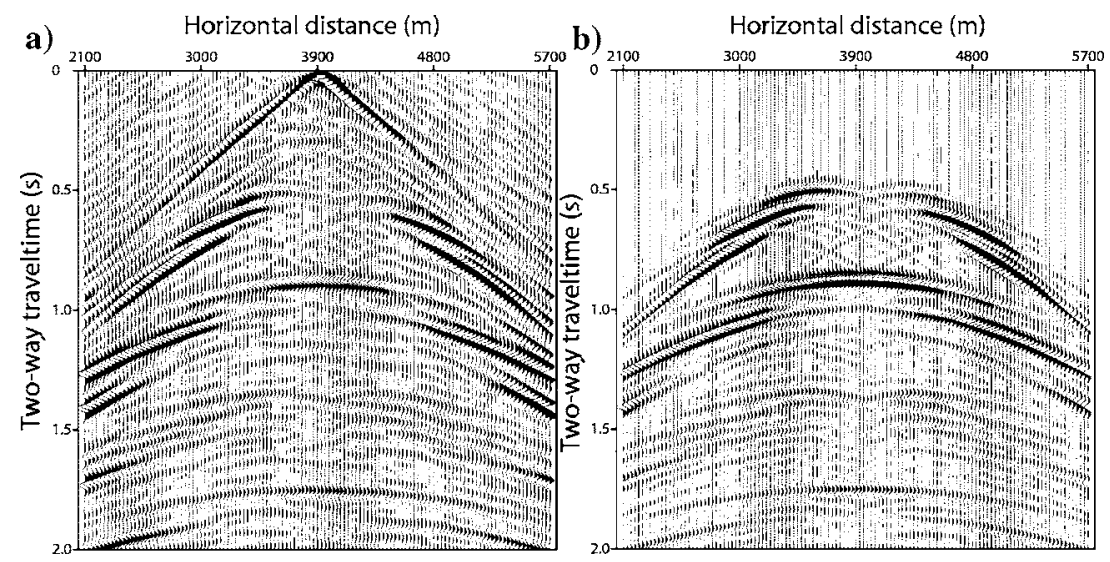

Figure 15. (a) Summed panels from Figure 14a and b after muting the acausal part. This represents the reconstructed vertical particle velocity $G_{3, \mathcal{3}}^{v, \tau}\left(\mathbf{x}_{A}, \mathbf{x}_{B}, t\right)$ resulting from a vertical traction source at horizontal position $x_{1}=3900 \mathrm{~m}$. (b) Directly modeled vertical particle velocity $G_{3,3}^{v, \tau}\left(\mathbf{x}_{A}, \mathbf{x}_{B}, t\right)$ resulting from a vertical traction source at horizontal position $x_{1}=3900 \mathrm{~m}$. All primary and multiply reflected events are reconstructed. illustrate the reconstruction of the reflection response $G_{p, q}^{v, \tau}\left(\mathbf{x}_{A}, \mathbf{x}_{B}, t\right)$ using equation 6 . Because we use a $2 \mathrm{D}$ model and vertical polarization, the subscripts $p$ and $q$ take only the value 1 or 3 ; the subscript $K$ can take the value 0 or 2 (for $\mathrm{P}$-wave sources and SV-wave sources, respectively). The model consists of two layers with two lenses embedded in the first layer. There were 240 receivers at the free surface, spaced every $15 \mathrm{~m}$ from $2100 \mathrm{~m}$ to $5700 \mathrm{~m}$. We used subsurface sources with random depth coordinates between $700 \mathrm{~m}$ and $800 \mathrm{~m}$. In the horizontal direction, the subsurface sources were distributed every $21 \mathrm{~m}$ between $2100 \mathrm{~m}$ and $5691 \mathrm{~m}$. Using this model, at each subsurface source position we generate transmission responses $G_{p, K}^{v, \phi}\left(\mathbf{x}_{A}, \mathbf{x}, t\right)$ from separate $\mathrm{P}$ - and $\mathrm{SV}$-wave sources $(K=0$ and $K=2$, respectively) using the finite element modeling code. Figures $13 \mathrm{a}$ and $\mathrm{b}$ show recorded vertical particle-velocity panels from subsurface P- and $\mathrm{SV}$-wave sources respectively. According to equation 6 , we can use these responses to reconstruct the observed free-surface vertical particle velocity that results from a vertical traction source at the free surface $\left[G_{3,3}^{v, \tau}\left(\mathbf{x}_{A}, \mathbf{x}_{B}, t\right)\right]$. To do this, we need to correlate the recorded responses from P- and SV-wave sources separately. First, we extract one master trace (for example, the trace at horizontal position $3900 \mathrm{~m}$ ) from the panel in Figure 13a and correlate it with all traces in the panel. The result of this operation represents the term $G_{3,0}^{v, \phi}\left(\mathbf{x}_{A}, \mathbf{x}, t\right) * G_{3,0}^{v, \phi}\left(\mathbf{x}_{B}, \mathbf{x},-t\right)$ for variable $\mathbf{x}_{A}$ and fixed $\mathbf{x}_{B}$ and $\mathbf{x}$. We repeat this process for all the subsurface P-wave sources, and just as we did in the acoustic case, we sum the individual results. The result is shown in Figure 14a and represents the first part (i.e., for $K=0$ ) of the integral in the right-hand side of equation 6 for variable $\mathbf{x}_{A}$ and fixed $\mathbf{x}_{B}$. As a second step, we perform the same procedure for all responses recorded at the surface from SV-wave sources in the subsurface (like the one in Figure 13b). The result is the second part (i.e., for $K=2$ ) of the integral in the right-hand side of equation 6 (see Figure 14b).

Finally, to reconstruct $G_{3,3}^{v, \tau}\left(\mathbf{x}_{A}, \mathbf{x}_{B}, t\right)$, we sum the panels from Figures $14 \mathrm{a}$ and $\mathrm{b}$, weighted by $2 / \rho c_{p}$ and $2 / \rho c_{s}$, respectively. The result (after muting the acausal part) is shown in Figure 15a. For comparison, Figure 15b shows the directly modeled vertical particle velocity resulting from the vertical traction source. Note that we have removed the direct waves and the surface waves from the directly modeled vertical particle velocity, and the reconstructed vertical particle velocity is shown as it is obtained from equation 6. Because no subsurface sources existed close to the surface, no surface waves are reconstructed. Apart from that, comparing Figure 15a and b, we see that all the reflection events are reconstructed, including the free-surface multiple arrivals. Just 


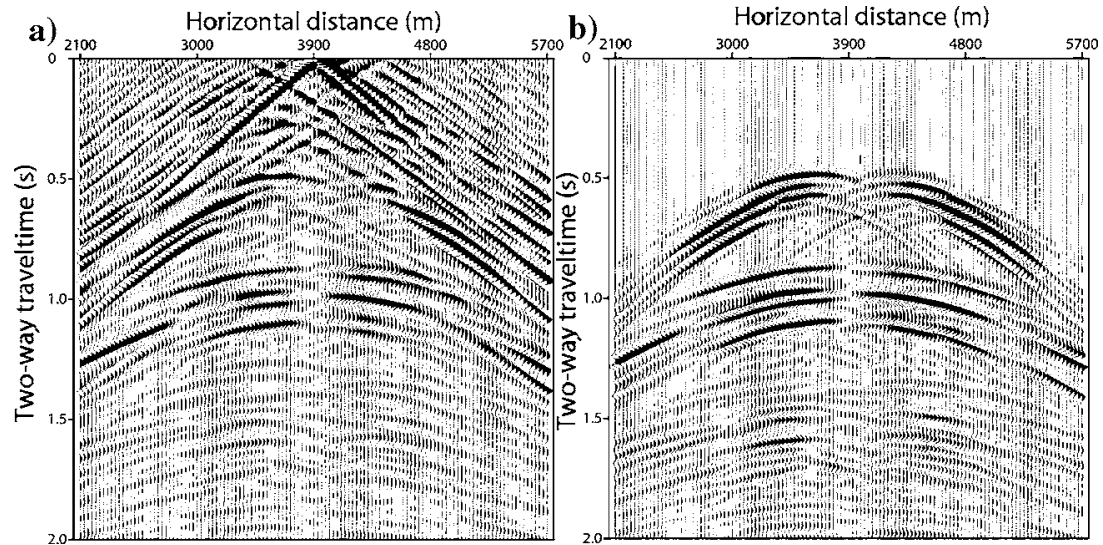

Figure 16. (a) A reconstructed vertical particle velocity $G_{3,1}^{v, \tau}\left(\mathbf{x}_{A}, \mathbf{x}_{B}, t\right)$ resulting from a horizontal traction source at horizontal position $x_{1}=3900 \mathrm{~m}$. (b) Directly modeled vertical particle velocity $G_{3,1}^{v, \tau}\left(\mathbf{x}_{A}, \mathbf{x}_{B}, t\right)$ resulting from a horizontal traction source at horizontal position $x_{1}=3900 \mathrm{~m}$. All primary and multiply reflected events are reconstructed.

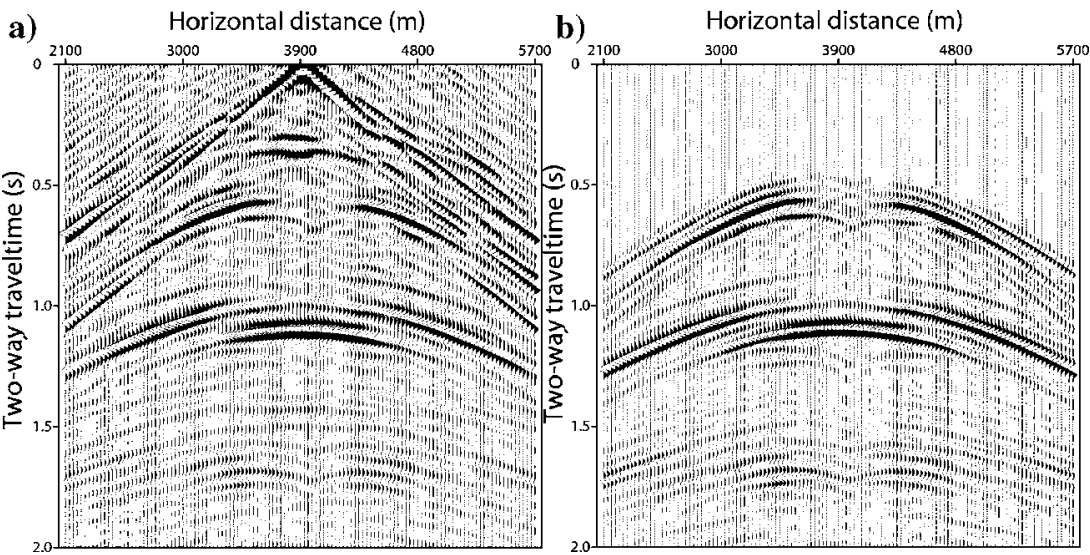

Figure 17. (a) A reconstructed horizontal particle velocity $G_{1,1}^{v, \tau}\left(\mathbf{x}_{A}, \mathbf{x}_{B}, t\right)$ resulting from a horizontal traction source at horizontal position $x_{1}=3900 \mathrm{~m}$. (b) Directly modeled horizontal particle velocity $G_{1,1}^{v} \tau\left(\mathbf{x}_{A}, \mathbf{x}_{B}, t\right)$ resulting from a horizontal traction source at horizontal position $x_{1}=3900 \mathrm{~m}$. All primary and multiply reflected events are reconstructed.

subsurface. In the extreme case of only a few sources, the reflection response cannot be reconstructed correctly. If after reconstruction the reflection responses are subsequently migrated, the subsurface is imaged accurately even for the case of only a few sources in the subsurface. An alternative way to obtain an image of the subsurface is to apply shot-profile migration directly to the transmission responses. The results from both migration paths are identical.

For the elastic case, we showed how to reconstruct the multicomponent reflection response that is, the Green's function observed at the free surface and resulting from a source at the free surface. The reconstruction is achieved from the crosscorrelation of Green's functions measured at the surface and resulting from separate $\mathrm{P}$ - and $\mathrm{S}$-wave transient sources in the subsurface (transmission responses).

\section{ACKNOWLEDGMENTS}

This research is supported by The Netherlands Research Centre for Integrated Solid Earth Sciences ISES and by the Technology Foundation STW, applied science division of NWO, and the technology program of the Ministry of Economic Affairs (grant DTN.4915). We would like to thank J. F. Zhang for providing the finite element code. Deyan Draganov would like to thank his colleagues Nikoletta Filippidou and Petar Angelov for their advice.

\section{REFERENCES}

Artman, B., 2006, Imaging passive seismic data: Geophysics, this issue.

Artman, B., D. Draganov, C. P. A. Wapenaar, and B Biondi, 2006, Direct migration of passive seismic data: 66th Annual International Conference and Exhibition, EAGE, Extended Abstracts, P075.

as it was in the acoustic case, the randomness of the sources in depth greatly weakened the ghost event that resulted from the reflector below the sources. At the same time, the true reflection from the deep layer is reconstructed correctly.

In Figures 16 and 17, we also compare the simulated and the directly modeled reflection responses for $G_{3,1}^{v, \tau}\left(\mathbf{x}_{A}, \mathbf{x}_{B}, t\right)$ (Figure 16) and for $G_{1, \tau}^{v, \tau}\left(\mathbf{x}_{A}, \mathbf{x}_{B}, t\right)$ (Figure 17). From those results we observe that such components of the reflection response also are reconstructed correctly after crosscorrelation.

\section{CONCLUSIONS}

We have shown that in the presence of a distribution of sources in the subsurface, we can reconstruct the reflection response of an inhomogeneous lossless medium by crosscorrelating the transmission responses measured at the surface. We can achieve this using two different processing schemes, depending on the type of subsurface sources: transient sources with distinct excitation times or noise sources acting simultaneously. The reconstructed reflection response strongly depends on the quantity of the sources present in the
Bakulin, A., and R. Calvert, 2004, Virtual source: New method for imaging and 4D below complex overburden: 74th Annual International Meeting, SEG, Expanded Abstracts, 2477-2480.

, 2006, The virtual source method: Theory and case study: Geophysics, this issue.

Campillo, M., and A. Paul, 2003, Long-range correlations in the diffuse seismic coda: Science 299, 547-549.

Claerbout, J. F., 1968, Synthesis of a layered medium from its acoustic transmission response: Geophysics, 33, 264-269.

, 1971, Toward a unified theory of reflector mapping: Geophysics, 36 467-481.

Derode, A., E. Larose, M. Tanter, J. de Rosny, A. Tourin, M. Campillo, and M. Fink, 2003, Recovering the Green's function from field-field correlations in an open scattering medium: Journal of the Acoustic Society of America, 113, 2973-2976.

Draganov, D., C. P. A. Wapenaar, and J. Thorbecke, 2004, Passive seismic imaging in the presence of white noise sources: The Leading Edge, 23, 889-892.

Lobkis, O. I., and R. L. Weaver, 2001, On the emergence of the Green's function in the correlations of a diffuse field: Journal of the Acoustic Society of America, 110,3011-3017.

Rickett, J., 1996, The effects of lateral velocity variations and ambient noise source location on seismic imaging by cross-correlation: Stanford Exploration Project Report, 93, 139-151.

Rickett, J., and J. F. Claerbout, 1996, Passive seismic imaging applied to synthetic data: Stanford Exploration Project Report, 92, 87-94.

Roux, P., K. G. Sabra, W. A. Kuperman, and A. Roux, 2005, Ambient noise 
cross correlation in free space: Theoretical approach: Journal of the Acoustic Society of America, 117, 79-84.

Sabra, K. G., P. Gerstoft, P. Roux, W. A. Kuperman, and M. C. Fehler, 2005 , Extracting time-domain Green's function estimates from ambient seismic noise: Geophysical Research Letters, 32, doi: 10.1029/2004GL021862.

Schuster, G. T., 2001, Theory of daylight/interferometric imaging: Tutorial: 63rd Annual International Conference and Exhibition, EAGE, Extended Abstracts, A-32.

Shapiro, N. M., and M. Campillo, 2004, Emergence of broadband Rayleigh waves from correlations of the ambient seismic noise: Geophysical Research Letters, 31, L07614, doi: 10.1029/2004 GL019491.

Shapiro, N. M., M. Campillo, L. Stehly, and M. H. Ritzwoller, 2005, Highresolution surface wave tomography from ambient seismic noise: Science, 307, 1615-1618.

Snieder, R., 2004, Extracting the Green's function from the correlation of coda waves: A derivation based on stationary phase: Physical Review E,
69, doi: 10.1103/Phys Rev E. 69.046610.

van Tiggelen, B. A., 2003, Green function retrieval and time reversal in a disordered world: Physical Review Letters, 91, doi: 10.1103/Phys Rev Lett. 91.243904

Wapenaar, C. P. A., and J. Fokkema, 2006, Green's functions representations for seismic interferometry: Geophysics, this issue.

Wapenaar, C. P. A., J. W. Thorbecke, and D. Draganov, 2004, Relations between reflection and transmission responses of 3-D inhomogeneous media: Geophysical Journal International, 156, 179-194.

Wapenaar, C. P. A., J. W. Thorbecke, D. Draganov, and J. T. Fokkema, 2002, Theory of acoustic daylight imaging revisited: 72nd Annual International Meeting, SEG, Expanded Abstracts, 2269-2272.

Weaver, R. L., and O. I. Lobkis, 2001, Ultrasonics without a source: Thermal fluctuation correlations at MHz frequencies: Physical Review Letters, 87, doi: 10.1103/Phys Rev Lett. 87.134301. 